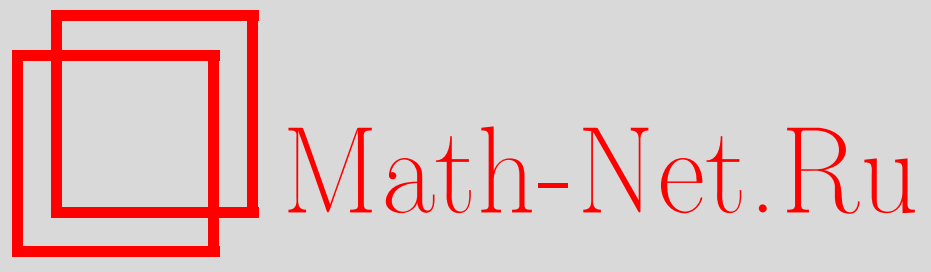

В. В. Грушин, С. Ю. Доброхотов, Подстановка Пайерлса и операторный метод Маслова, Матем. заметки, 2010, том 87, выпуск 4, 554-571

DOI: https://doi.org/10.4213/mzm8699

Использование Общероссийского математического портала Math-Net.Ru подразумевает, что вы прочитали и согласны с пользовательским соглашением http://www .mathnet.ru/rus/agreement

Параметры загрузки:

IP : 34.229 .45 .116

26 апреля 2023 г., 16:12:13

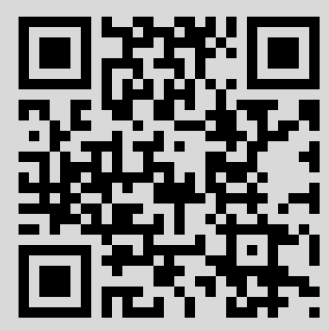


Том 87 выпуск 4 апрель 2010

УДК 517.9

\section{Подстановка Пайерлса \\ и операторный метод Маслова}

\section{В. В. Грушин, С. Ю. Доброхотов}

Рассматривается периодический оператор Шрёдингера в постоянном магнитном поле с векторным потенциалом $A(x)$. Подстановка Пайерлса представляет собой вариант адиабатического приближения для уравнений квантовой механики с быстро меняющимися электрическими потенциалами и слабыми магнитными полями, что в подходящих обезразмеренных переменных позволяет написать псевдодифференциальное уравнение для новой вспомогательной функции: $\mathscr{E}^{\nu}\left(-i \mu \partial_{x}, x\right) \phi=E \phi, \mathscr{E}^{\nu}-$ соответствующий энергетический уровень некоторого вспомогательного оператора Шрёдингера, который предполагается невырожденным, $\mu$ - малый параметр. В статье с помощью операторного метода В.П. Маслова показывается, что в случае постоянного магнитного поля такая редукция в любом порядке теории возмущений приводит к уравнению $\mathscr{E}^{\nu}(\widehat{P}, \mu) \phi=E \phi$, с оператором $\mathscr{E}^{\nu}(\widehat{P}, \mu)$, представленным как функция, зависящая только от операторов длинных импульсов $\widehat{P}_{j}=-i \mu \partial_{x_{j}}+A_{j}(x)$.

Библиография: 13 названий.

1. Алгебра длинных импульсов. Подстановка Пайерлса представляет собой вариант адиабатического приближения для уравнений квантовой механики с быстро меняющимися электрическими потенциалами и слабыми магнитными полями. Рассмотрим случай периодического оператора Шрёдингера в постоянном магнитном поле, когда в трехмерном случае в подходящих безразмерных переменных стационарное уравнение Шрёдингера можно записать в виде

$$
\left(-i \mu \frac{\partial}{\partial x}+A(x)\right)^{2} \Psi+v\left(\frac{x}{\mu}\right) \Psi=E \Psi,
$$

где $v(y)$ - потенциал, периодический на решетке $\Gamma$, задаваемой базисными векторами периодов $a_{1}, a_{2}, a_{3}: v\left(y+a_{j}\right)=v(y)$. Параметр $\mu$ считается малым, он характеризует малость реальных периодов исходного потенциала $v(y)$, векторный потенциал $A(x)$ выбран в симметричной калибровке: $A(x)=(1 / 2) B \times x$, слабость магнитного поля $B$ означает, что $B$ не зависит от параметра $\mu,|B|=O(1)$. Напомним, что подстановка Пайерлса состоит в следующей процедуре [1]. Рассмотрим семейство

Работа выполнена при поддержке Российского фонда фундаментальных исследований (грант № 08-01-00726) и Министерства образования и науки РФ (грант № 2.1.1/4540). 
зависящих от параметров квазиимпульса $P$ задач о блоховских функциях для оператора $-\Delta_{y}+v(y)$ :

$$
\left(-\Delta_{y}+v(y)\right) \chi=\mathscr{E}(P) \chi, \quad \chi\left(y+a_{j}, P\right)=e^{i P \cdot a_{j}} \chi(y, P) .
$$

При каждом $P \in \mathbb{R}^{3}$ спектр этой задачи дискретен, при этом функции $\chi(y, P)$ можно выбрать таким образом, что собственные значения $\mathscr{E} \nu(P)$, занумерованные индексом $\nu$, будут непрерывными функциями, периодическими на обратной решетке $\Gamma^{*}$, заданной базисными векторами $b_{1}, b_{2}, b_{3}$, где $a_{j} \cdot b_{k}=2 \pi \delta_{j k}$. Функции $\mathscr{E} \nu(P)$ часто называют термами, а равенства $\omega=\mathscr{E}^{\nu}(P)$ - дисперсионным соотношением. В дальнейшем будем предполагать, что выполнено следующее.

УсловиЕ (i). Для некоторого фиксированного $\nu$ соответствующее собственное значение $\mathscr{E}^{\nu}(P)$ не вырождено для любого $P$ :

$$
\left|\mathscr{E}^{\nu}(P)-\mathscr{E}^{s}(P)\right|>\delta>0
$$

для всех $s \neq \nu, P \in \mathbb{R}^{3}$.

В таком случае $\mathscr{E} \nu(P)$ - гладкая функция, периодическая с периодами $b_{j}$, при этом (см. [2]) соответствующую блоховскую функцию $\chi^{\nu}(y, P)$ можно выбрать так, что она будет гладкой функцией, периодической по переменным $P$ с периодами $b_{j}$, т.е. периодической относительно обратной решетки $\Gamma^{*}$. Мы также будем считать, что при фиксированном $P$ функция $\chi^{\nu}(y, P)$ нормирована:

$$
\left(\chi^{\nu}(y, P), \chi^{\nu}(y, P)\right)_{y}=1
$$

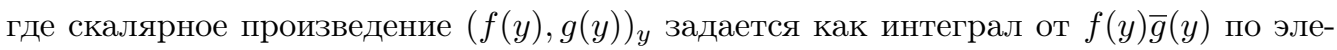
ментарной ячейке $Q$ решетки $\Gamma$ (например, по замкнутому параллелепипеду, построенному на векторах $a_{j}$ ). Подстановка Пайерлса в классической механике состоит в замене вектора квазиимпульса $P$ на длинный классический импульс $P(p, x)=$ $p+A(x)$, что приводит к появлению эффективного гамильтониана $H^{\nu}=\mathscr{E}^{\nu}(p+A(x))$, задающего соответствующую гамильтонову систему в фазовом пространстве с координатами $(p, x)$. “Квантовая" подстановка Пайерлса состоит в замене классического импульса $p$ на оператор $\widehat{p}=-i \mu \partial / \partial x$, что позволяет написать псевдодифференциальное редуцированное уравнение для новой вспомогательной функции $\varphi$ :

$$
\widehat{L}^{\nu} \varphi=E \varphi, \quad L^{\nu}(p, x)=\mathscr{E}^{\nu}(p+A(x))+O(\mu) .
$$

Поскольку операторы $\widehat{p}$ и $x$ не коммутируют, то возникает вопрос о "правильном" построении теории возмущений. Этому вопросу, который сформулирован, в частности, в книге [1] посвящено много публикаций. На операторном уровне он по существу (без псевдодифференциальных операторов) был решен в любом порядке теории возмущений в работе [3] для любого, в том числе и непостоянного магнитного поля. Используемая в [3] техника и формулы имеют достаточно сложную структуру. Сравнительно недавно построение редуцированного уравнения, основанного на псевдодифференциальных операторах в вейлевской форме, с соответствующими обоснованиями было проведено в работе [2]. В этой заметке мы хотим показать, что в случае постоянного поля оператор $\widehat{L}^{\nu}(p, x)$ в редуцированном уравнении в любом порядке теории возмущений имеет вид функции, зависящей только от операторов длинных импульсов $\widehat{P}_{j}=-i \mu \partial / \partial x_{j}+A_{j}(x)$, при этом такая редукция к уравнению (1.3) может быть произведена относительно просто с помощью операторного 
метода Маслова (см. [4]). Задача (1.1), как было отмечено уже в [1], имеет алгебраическую природу. Операторы “длинных импульсов" $\widehat{P}_{j}$ и координатные операторы $x_{j}$ являются образующими алгебры Гейзенберга с коммутационными соотношениями

$$
\left[\widehat{P}_{j}, \widehat{P}_{k}\right]=2 i \mu q_{j k}, \quad\left[\widehat{P}_{j}, x_{k}\right]=-i \mu \delta_{j k}, \quad\left[x_{j}, x_{k}\right]=0,
$$

где константы $q_{j k}$ - это коэффициенты линейных функций

$$
A_{j}(x)=\sum q_{j k} x_{k}
$$

В нашем случае $q_{12}=-B_{3} / 2, q_{13}=B_{2} / 2, q_{23}=-B_{1} / 2$ и матрица $\left(q_{j k}\right)$ кососимметрична. Гамильтониан $\widehat{H}$ имеет вид $\widehat{H}=\widehat{P}^{2}+v(x / \mu)$. Поскольку операторы $\widehat{P}_{j}$ не коммутируют между собой, то нужно задавать их упорядочение, для определенности будем считать, что оператор $\widehat{P}_{1}$ действует первым, $\widehat{P}_{2}-$ вторым, $\widehat{P}_{3}-$ третьим и, используя обозначения Фейнмана-Маслова [4], можно писать

$$
\widehat{P}_{1}^{1}, \quad \widehat{P}_{2}, \quad \widehat{P}_{3}
$$

Это упорядочение не существенно для определения $\widehat{H}$, но играет важную роль для дальнейшего. Поскольку действие операторов $\widehat{P}_{1}, \widehat{P}_{2}, \widehat{P}_{3}$ всегда в дальнейшем будет упорядочено указанным образом, цифры над операторами мы будем в дальнейшем опускать. Обобщая предложенный в [5]-[7] подход (“обобщенный адиабатический принцип") с учетом вхождения операторов дифференцирования и умножения на (медленные) переменные $x_{j}$ в гамильтониан $\widehat{H}$ только через операторы длинных импульсов, адиабатическое приближение можно сформулировать следующим образом. Будем искать некоторое частное решение - волновую функцию $\Psi$ в виде

$$
\Psi=\widetilde{\Psi}(x, y)=\widehat{\chi}^{\nu} \varphi
$$

где

$$
\widehat{\chi}^{\nu}=\chi^{\nu}\left(y, \widehat{P}_{1}^{1}, \widehat{P}_{2}^{2}, \widehat{P}_{3}^{3}, \mu\right)
$$

считая, что $y=x / \mu, \chi^{\nu}(y, P, \mu)$ - гладко зависящая от $y$ и $P$ функция, периодическая по $y$ с периодами $a_{j}$, причем новая неизвестная функция $\varphi$ удовлетворяет уравнению (обобщенная подстановка Пайерлса)

$$
\widehat{L}^{\nu} \varphi=E^{\nu} \varphi
$$

с оператором

$$
\widehat{L}^{\nu}=L^{\nu}\left(\widehat{P}_{1}^{1}, \widehat{P}_{2}, \widehat{P}_{3}^{3}, \mu\right),
$$

представленным как функция, зависящая только от операторов $\widehat{P}_{j}$ и $\mu$. Операторы $\widehat{\chi}^{\nu}$ называются восстанавливающими или сплетающими. Операторы $\widehat{\chi}^{\nu}, \widehat{L}^{\nu}$ задаются символами $\chi^{\nu}(y, P, \mu), L^{\nu}(P, \mu)$, гладко зависящими от своих переменных и разлагающимися в асимптотические ряды по малому параметру $\mu$ :

$$
L^{\nu}(P, \mu)=L_{0}^{\nu}(P)+\mu L_{1}^{\nu}(P)+\cdots, \quad \chi^{\nu}(y, P, \mu)=\chi_{0}^{\nu}(y, P)+\mu \chi_{1}^{\nu}(y, P)+\cdots .
$$

О том, каким классам принадлежат соответствующие символы, мы скажем ниже. Пользуясь соотношениями (1.4) и (1.5), уравнение (1.1) можно записать в виде операторного соотношения

$$
\left(\left(\widehat{P}-i \frac{\partial}{\partial y}\right)^{2}+v(y)\right) \chi^{\nu}(y, \widehat{P}, \mu)=\chi^{\nu}(y, \widehat{P}, \mu) L^{\nu}(\widehat{P}, \mu) .
$$


Основная идея дальнейших вычислений состоит в следующем. Сначала от операторного уравнения (1.7) нужно перейти к уравнению для символов $\chi_{k}^{\nu}(y, P)$ и $L_{k}^{\nu}(P)$ операторов $\widehat{\chi}_{k}^{\nu}$ и $\widehat{L}_{k}^{\nu}$. Затем мы находим коэффициенты разложений (1.6) с помощью рекуррентных соотношений, которые получаются путем разложения левой и правой частей этого уравнения в асимптотические ряды по параметру $\mu$ и приравнивания коэффициентов при одинаковых степенях параметра $\mu$. Мы ограничимся только анализом уравнений нулевого и первого приближений, хотя данным методом можно находить и следующие приближения, что, однако, привело бы к достаточно громоздким вычислениям. Имеют место следующие формулы композиции операторов и ее асимптотического разложения, которые можно найти в [8; с. 175-176] и которые мы также будем обсуждать в п. 3 данной статьи. Рассмотрим два оператора

$$
\widehat{a}_{1}=a_{1}\left(\widehat{P}_{1}, \widehat{P}_{2}, \widehat{P}_{3}\right), \quad \widehat{a}_{2}=a_{2}\left(\widehat{P}_{1}, \widehat{P}_{2}, \widehat{P}_{3}\right)
$$

с символами $a_{1}\left(P_{1}, P_{2}, P_{3}\right), a_{2}\left(P_{1}, P_{2}, P_{3}\right)$ и их композицию - оператор

$$
\widehat{b} \equiv b\left(\widehat{P}_{1}, \widehat{P}_{2}, \widehat{P}_{3}\right)=\widehat{a}_{2} \widehat{a}_{1} .
$$

Построим операторы

$$
\begin{gathered}
\widehat{\mathbf{l}}_{1}=P_{1}+\mu l_{1}, \quad l_{1}=-i B_{3} \frac{\partial}{\partial P_{2}}+i B_{2} \frac{\partial}{\partial P_{3}}, \\
\widehat{\mathbf{l}}_{2}=P_{2}+\mu l_{2}, \quad l_{2}=-i B_{1} \frac{\partial}{\partial P_{3}}, \quad \widehat{\mathbf{l}}_{3}=P_{3} .
\end{gathered}
$$

Тогда согласно [4], [8] по крайней мере формально можно записать символ оператора $\widehat{a}_{2} \widehat{a}_{1}$ в виде

$$
b\left(P_{1}, P_{2}, P_{3}, \mu\right)=a_{2}\left(\widehat{1}_{1}, \stackrel{2}{\mathbf{l}}_{2}, \widehat{3}_{3}\right) a_{1}\left(P_{1}, P_{2}, P_{3}\right) \equiv a_{2}\left(\stackrel{2}{P_{1}}+\mu \stackrel{1}{\widehat{l}_{1}}, \stackrel{2}{P_{2}}+\mu \stackrel{1}{\widehat{l}_{2}}, \stackrel{2}{P_{3}}\right) a_{1}\left(P_{1}, P_{2}, P_{3}\right) .
$$

Последнее из этой цепочки равенств справедливо в силу того факта, что операторы $\widehat{l}_{1}, P_{1}$ и $\widehat{l}_{2}, P_{2}$ коммутируют. Поэтому уравнение для символов $\chi, L$ имеет вид

$$
\begin{aligned}
& \left(\left(\stackrel{2}{P}_{1}+\mu \widehat{\widehat{l}}_{1}-i \frac{\partial}{\partial y_{1}}\right)^{2}+\left(\stackrel{2}{P}_{2}+\mu \widehat{\widehat{l}}_{2}-i \frac{\partial}{\partial y_{2}}\right)^{2}+\left(\stackrel{2}{P}_{3}-i \frac{\partial}{\partial y_{3}}\right)^{2}+v(y)\right) \chi^{\nu}(y, P, \mu) \\
& =\chi^{\nu}\left(y, \stackrel{2}{P}_{1}+\mu \widehat{l}_{1}, \stackrel{2}{P}_{2}+\mu \widehat{l}_{2}, \stackrel{2}{P}_{3}, \mu\right) L^{\nu}(P, \mu) .
\end{aligned}
$$

Теперь мы можем написать разложения по параметру $\mu$ и приравнять коэффициенты при разных степенях $\mu$ в левой и правой частях этого равенства. Это дает цепочку уравнений для определения коэффициентов $\chi^{\nu}$ и $L^{\nu}$.

Прежде чем выписать уравнения из этой цепочки отметим следующее. Хотя последние равенства следуют из общей операторной теории Маслова [4], [8], тем не менее, для полноты изложения мы приведем их доказательства в п. 3 в форме, вероятно, более привычной для специалистов в области псевдодифференциальных операторов. Для замкнутости изложения в п. 2 мы приведем некоторые вспомогательные для п. 3 утверждения, касающиеся метода стационарной фазы. В нулевом приближении получаем уравнение

$$
\left(\left(P-i \partial_{y}\right)^{2}+v(y)\right) \chi_{0}^{\nu}(y, P)=\mathscr{E}^{\nu}(P) \chi_{0}^{\nu}(y, P),
$$


и мы можем взять

$$
\chi_{0}^{\nu}(y, P)=e^{-i P \cdot y} \chi^{\nu}(y, P),
$$

где $\chi^{\nu}(y, P)$ - соответствующая блоховская функция из $(1.2)$, а $L_{0}^{\nu}(P)=\mathscr{E}(P)$. В результате мы получаем редуцированное уравнение в нулевом приближении:

$$
\mathscr{E}^{\nu}(\widehat{P}) \varphi=E \varphi
$$

что согласуется с (1.3). В силу периодичности на обратной решетке $\Gamma^{*}$ блоховской функции $\chi^{\nu}(y, P)$ для $\chi_{0}^{\nu}(y, P)$ имеем

$$
\chi_{0}^{\nu}\left(y, P+b_{j}\right)=e^{-i b_{j} \cdot y} \chi_{0}^{\nu}(y, P) .
$$

Отсюда легко выводятся равенства

$$
\frac{\partial \chi_{0}^{\nu}}{\partial P}\left(y, P+b_{j}\right)=e^{-i b_{j} \cdot y} \frac{\partial \chi_{0}^{\nu}}{\partial P}(y, P), \quad \frac{\partial \chi_{0}^{\nu}}{\partial y}\left(y, P+b_{j}\right)=e^{-i b_{j} \cdot y}\left(\frac{\partial}{\partial y}-i b_{j}\right) \chi_{0}^{\nu}(y, P)
$$

для $j=1,2,3$.

Для вычисления следующей поправки составляем уравнение, приравнивая коэффициенты при степенях $k$ параметра $\mu$ :

$$
\left(\left(P-i \partial_{y}\right)^{2}+v(y)-\mathscr{E}^{\nu}(P)\right) \chi_{k}^{\nu}(y, P)=F_{k}(y, P)+L_{k}^{\nu}(P) \chi_{0}^{\nu}(y, P),
$$

где $F_{k}$ выражается только через $\chi_{0}^{\nu}, \ldots, \chi_{k-1}^{\nu}$ и $L_{0}^{\nu}, \ldots, L_{k-1}^{\nu}$ и их производные, при этом производные $\partial / \partial y_{m}$ входят только в виде комбинаций $\left(P_{m}-i \partial / \partial y_{m}\right)$. В частности,

$$
\begin{aligned}
F_{1}(y, P)=- & i \widetilde{q}_{12} \partial_{P_{1}} \chi_{0}^{\nu} \partial_{P_{2}} L_{0}^{\nu}-i \widetilde{q}_{13} \partial_{P_{1}} \chi_{0}^{\nu} \partial_{P_{3}} L_{0}^{\nu}-i \widetilde{q}_{23} \partial_{P_{2}} \chi_{0}^{\nu} \partial_{P_{3}} L_{0}^{\nu} \\
& -2\left(-i \widetilde{q}_{12}\left(P_{1}-i \partial_{y_{1}}\right) \partial_{P_{2}}-i \widetilde{q}_{13}\left(P_{1}-i \partial_{y_{1}}\right) \partial_{P_{3}}\right. \\
& -i \widetilde{q}_{23}\left(P_{2}-i \partial_{y_{2}}\right) \partial_{P_{3}} \chi_{0}^{\nu}(y, P)
\end{aligned}
$$

где $\widetilde{q}_{j k}=q_{k j}-q_{j k}$. Используя равенства (1.10), нетрудно доказать равенство

$$
F_{1}\left(y, P+b_{j}\right)=e^{-i b_{j} \cdot y} F_{1}(y, P) .
$$

Поскольку в левой части (1.11) стоит оператор, который при фиксированном $P$ является фредгольмовым в соответствующих соболевских пространствах периодических относительно решетки Г функций (т.е. фредгольмовым оператором из $\mathscr{H}_{\mathrm{per}}+2$ в $\mathscr{H}_{\text {per }}^{s}$ ), что следует из общей теории эллиптических операторов на компактных многообразиях (см., например, [9]), то условие разрешимости уравнения (1.11) состоит в ортогональности правой части решению однородного уравнения

$$
L_{1}^{\nu}(P)=-\left(F_{1}(y, P), \chi_{0}^{\nu}(y, P)\right)_{y} .
$$

Используя равенства (1.10) и (1.13), получим

$$
L_{1}^{\nu}\left(P+b_{j}\right)=L_{1}^{\nu}(P) .
$$

Используя в свою очередь это равенство, найдем, что

$$
F_{1}\left(y, P+b_{j}\right)+L_{1}^{\nu}\left(P+b_{j}\right) \chi_{0}^{\nu}\left(y, P+b_{j}\right)=e^{-i b_{j} \cdot y}\left(F_{1}(y, P)+L_{1}^{\nu}(P) \chi_{0}^{\nu}(y, P)\right) .
$$


Для того чтобы функция $\chi_{1}^{\nu}(y, P)$ находилась из уравнения (1.11) единственным образом, наложим дополнительное условие ее ортогональности к $\chi_{0}^{\nu}(y, P)$ :

$$
\left(\chi_{1}^{\nu}(y, P), \chi_{0}^{\nu}(y, P)\right)_{y}=0 .
$$

Поскольку в левой части уравнения (1.11) производные $\partial / \partial y_{j}$ также входят только в виде комбинаций $\left(P_{j}-i \partial / \partial y_{j}\right)$, то из (1.16) немедленно следует, что

$$
\chi_{1}^{\nu}\left(y, P+b_{j}\right)=e^{-i b_{j} \cdot y} \chi_{1}^{\nu}(y, P) .
$$

Ясно, что аналогичным образом можно получать и дальнейшие приближения из следующих рекуррентных соотношений (1.11). Более того, учитывая описанные выше свойства функций $F_{k}$, индукцией по номеру приближения нетрудно установить равенства

$$
L_{k}^{\nu}\left(P+b_{j}\right)=L_{k}^{\nu}(P), \quad \chi_{k}^{\nu}\left(y, P+b_{j}\right)=e^{-i b_{j} \cdot y} \chi_{k}^{\nu}(y, P), \quad \chi_{k}^{\nu}\left(y+a_{l}, P\right)=\chi_{k}^{\nu}(y) .
$$

В результате в любом порядке теории возмущений получается редуцированное уравнение:

$$
\left(\mathscr{E} \nu\left(\widehat{P}_{1}, \widehat{P}_{2}, \widehat{P}_{3}\right)+\mu L_{1}^{\nu}\left(\widehat{P}_{1}, \widehat{P}_{2}, \widehat{P}_{3}\right)+\cdots+\mu^{m} L_{m}^{\nu}\left(\widehat{P}_{1}, \widehat{P}_{2}, \widehat{P}_{3}\right)\right) \varphi=E \varphi
$$

где $L_{1}^{\nu}(P)$ определяется формулами (1.12) и (1.14).

Теперь дадим определение классов рассматриваемых символов.

ОПРЕДЕЛЕНИЕ 1.1. Пространство $S^{m}$ состоит из таких функций $f(P) \in \mathbb{R}_{P}^{3}$, что $f(P)$ и ее производные удовлетворяют неравенствам

$$
\left|f^{(\alpha)}(P)\right| \leqslant C_{\alpha}(1+|P|)^{m}
$$

с некоторыми константами $C_{\alpha}$ для всех мультииндексов $\alpha=\left(\alpha_{1}, \alpha_{2}, \alpha_{3}\right)$ с целыми неотрицательными $\alpha_{j}$.

В частности, $S^{0}$ состоит из всех ограниченных в $\mathbb{R}_{P}^{3}$ функций, имеющих ограниченные производные. Символ $L_{0}^{\nu}(P)$ как раз принадлежит $S^{0}$, поскольку он совпадает с $\mathscr{E} \nu(P)$, а $\mathscr{E} \nu(P)$ в силу сделанных предположений является гладкой периодической относительно решетки $\Gamma^{*}$ функцией. В $S^{m}$ можно ввести систему норм

$$
\|f(P)\|_{k, m}=\sum_{|\alpha| \leqslant k} \sup \frac{\left|f^{(\alpha)}(P)\right|}{(1+|P|)^{m}},
$$

в результате чего $S^{m}$ становится счетно-нормированным пространством. Мы будем писать, что

$$
f(P, h)=O\left(h^{N}\right) \quad \text { относителъно } S^{m},
$$

если каждая норма $\left\|h^{-N} f(P, h)\right\|_{k, m}$ ограничена в окрестности $h=0$.

ОПРЕДЕЛЕНИЕ 1.2. Функция $f(y, P) \in C^{\infty}\left(Q \times \mathbb{R}_{P}^{3}\right)$ принадлежит $S^{m}$, если выполняются оценки (1.21) с константами, не зависящими от $y$ при $y \in Q$.

Легко видеть, что функция $\chi_{0}^{\nu}$ как раз принадлежит $S^{0}$. Это следует из того, что она периодична относительно решетки $\Gamma$ по $y$, а функция $e^{i P \cdot y} \chi_{0}^{\nu}$ периодична относительно решетки $\Gamma^{*}$ по $P$, поскольку она совпадает с блоховской функцией $\chi^{\nu}(y, P)$. Функция $\chi^{\nu}(y, P)$ при $y \in Q$ полностью определяется своими значениями 
при $P \in Q^{*}$, где $Q$ и $Q^{*}$ - элементарные ячейки решеток $Г$ и $\Gamma^{*}$ соответственно, а на $Q \times Q^{*}$ она и ее производные ограничены, из чего следует, что функция $\chi_{0}^{\nu}(y, P)$ при $y \in Q$ имееет ограниченные производные по $P$. Тем самым, функция $\chi_{0}^{\nu}(y, P)$ принадлежит $S^{0}$. Аналогичное рассуждение показывает, что любая гладкая функция $f(y, P)$ на $Q \times \mathbb{R}_{P}^{3}$, для которой выполняется соотношение

$$
f\left(y, P+b_{j}\right)=e^{-i b_{j} \cdot y} f(y, P), \quad j=1,2,3,
$$

также принадлежит $S^{0}$. Соотношение (1.22) означает инвариантность $f(y, P)$ относительно преобразований

$$
\tau(b) f(y, P)=e^{i b \cdot y} f(y, P+b)
$$

для любых $b \in \Gamma^{*}$. Такие функции будем называть $\tau$-инвариантными. Таким образом, соотношение (1.22) означает, что функция $f(y, P)$ будет $\tau$-инвариантной.

Перейдем теперь к анализу символов первого приближения. Из (1.16) видим, что для правой части уравнения (1.11) при $k=1$ выполняется (1.22). Как уже отмечалось выше, из этого следует, что соотношение (1.22) будет выполняться и для решения $\chi_{1}^{\nu}(y, P)$. Действительно, из периодичности $\mathscr{E} \nu(P)$ относительно решетки $\Gamma^{*}$ следует, что оператор, стоящий в левой части уравнения (1.11) коммутирует с преобразованием $\tau(b)$ при $b \in \Gamma^{*}$ :

$\left(\left(P-i \partial_{y}\right)^{2}+v(y)-\mathscr{E}^{\nu}(P)\right) e^{i b \cdot y} u(y, P)=e^{i b \cdot y}\left(\left(P+b-i \partial_{y}\right)^{2}+v(y)-\mathscr{E}^{\nu}(P+b)\right) u(y, P)$.

Поэтому из (1.11), (1.15), (1.16) получим

$$
\begin{aligned}
& \left(\left(P-i \partial_{y}\right)^{2}+v(y)-\mathscr{E}^{\nu}(P)\right)\left(e^{i b \cdot y} \chi_{1}^{\nu}(y, P)\right) \\
& \quad=e^{i b \cdot y}\left(F_{1}(y, P+b)+L_{1}^{\nu}(P+b) \chi_{0}^{\nu}(y, P+b)\right)=F_{1}(y, P)+L_{1}^{\nu}(P) \chi_{0}^{\nu}(y, P) .
\end{aligned}
$$

Равенство (1.22) теперь следует из единственности решений уравнений (1.11) в классе периодических относительно решетки $\Gamma^{*}$ функций с условием ортогональности к $\chi_{0}^{\nu}(y, P)$. Фактически это рассуждение показывает, что из $\tau$-инвариантности правой части уравнения (1.11) вытекает $\tau$-инвариантность его решения.

Таким же образом по индукции устанавливаются равеннства (1.18). Бесконечную дифференцируемость присутствующих в них функций несложно доказать с помощью общей теории эллиптических операторов на компактных многообразиях (см., например, [9]). Для этого рассмотрим задачу: при фиксированном $P$ по заданной функции $f(y)$ и числу $d$ нужно найти функцию $u(y)$ и число $g$, удовлетворяющие уравнениям

$$
\left(\left(P-i \partial_{y}\right)^{2}+v(y)-\mathscr{E}^{\nu}(P)\right) u(y)-g \chi_{0}^{\nu}(y, P)=f(y), \quad\left(u(y), \chi_{0}^{\nu}(y, P)\right)_{y}=d .
$$

Так как отвечающий этой задаче оператор $A(P): \mathscr{H}^{s+2} \times C^{1} \rightarrow \mathscr{H}^{s} \times C^{1}$, где $C^{1}-$ одномерное комплексное пространство, обратим и $A(P)$ гладко зависит от параметров $P$, то обратный оператор $A^{-1}(P)$ также гладко зависит от параметров $P$. Взяв за $f$ функцию $F_{1}(y, P)$, мы получим, что решение $u(y, P, d)$ задачи $(1.23)$ гладко зависит от параметров $P$. Но $\chi_{1}^{\nu}(y, P)$ как раз и является решением задачи $(1.23)$ при $d=0$ и $g=L_{1}^{\nu}(P)$, следовательно, $\chi_{1}^{\nu}(y, P)$ является гладкой функцией от параметров $P$ со значениями в пространстве $\mathscr{H}^{s+2}$ при любом $s$. Теперь из стандартрых теорем вложения соболевских пространств вытекает, что $\chi_{1}^{\nu}(y, P)$ будет бесконечно дифференцируемой функцией по совокупности переменных. 
Аналогичным образом из рекуррентных соотношений (1.11) индукцией по $k$ доказываются равенства (1.18) и гладкость соответствующих функций. Тем самым, установлено следующее утверждение.

ТЕОРема 1.1. При выполнении условия (i) рекуррентные соотношения (1.11) имеют решения $\chi_{k}^{\nu}(y, P)$ и $L_{k}^{\nu}(P)$, принадлежсащие классу $S^{0}$ и удовлетворяющие соотношениям (1.18).

Теперь для любого целого $m \geqslant 0$ можно написать редуцированное уравнение в $m$-м приближении (1.19). Считая, что это равенство выполнено, построим функцию $\Psi(x)$ с помощью приближенного сплетающего оператора:

$$
\Psi(x)=\left.\left(\chi_{0}^{\nu}(y, \widehat{P})+\mu \chi_{1}^{\nu}(y, \widehat{P})+\cdots+\mu^{m} \chi_{m}^{\nu}(y, \widehat{P})\right)\right|_{y=x / \mu} \varphi(x) .
$$

Естественно, что уравнение (1.1) для такой функции будет выполняться лишь приближенно, и поэтому важным является вопрос о характере соответствующей невязки. Ответ дает следующее утверждение.

Теорема 1.2. Если выполнены условия теоремы 1.1, то функиия $\Psi(x)$, построенная с помощъю формуль (1.24), удовлетворяет соотношению

$$
\left(\left(-i \mu \partial_{x}+A(x)\right)^{2}+v(y)\right) \Psi-E \Psi+\left.\mu^{m+1} r_{m}(y, \widehat{P}, \mu)\right|_{y=x / \mu} \varphi(x)=0,
$$

где символ $r_{m}(y, \widehat{P}, \mu)$ принадлежит классу $S^{0}$ в смысле определения 1.2 , причем постоянные в неравенствах вида (1.21) для $r_{m}$ можно выбрать не зависящими от $0<\mu \leqslant 1$.

ДоКАЗАТЕЛЬСтво этого утверждения непосредственно следует из предыдущих рассуждений и оценки остаточного члена в формуле композиции операторов, которая рассматривается в п. 3 настоящей статьи.

Символ $r_{m}(y, \widehat{P}, \mu)$ также обладает свойством $(1.22)$, что вытекает из формулы композиции (1.8) и эквивалентной ей формулы (3.13).

Заметим также, что мы не указали класс, которому принадлежит $\varphi(x)$, нужно только, чтобы к таким функциям можно было бы применять операторы с символами класса $S^{0}$. Например, как это видно из п. 2 настоящей статьи, все предыдущие рассуждения справедливы, когда функция $\varphi$ принадлежит одному из классов $S^{k}$ (в определении 1.1 в этом случае переменные $P$ нужно заменить на $x$ ).

Некоторые следствия. Заметим, что если ось $x_{3}$ в исходном операторе выбрать направленной по вектору магнитного поля $B$, то оператор $\widehat{P}_{3}$ будет просто оператором дифференцирования, коммутирующим с $\widehat{P}_{1}, \widehat{P}_{2}$, к тому же не зависящими от переменной $x_{3}$. Поэтому по переменной $x_{3}$ в уравнении (1.19) можно сделать преобразование Фурье и получить для соответствующей функции $\widetilde{\varphi}\left(x_{1}, x_{2}, \xi_{3}\right)$, где $\xi_{3}-$ импульсная переменная, сопряженная $x_{3}$, уравнение

$$
\left(\mathscr{E}^{\nu}\left(\widehat{P}_{1}, \widehat{P}_{2}, \xi_{3}\right)+\mu^{m} L_{m}^{\nu}\left(\widehat{P}_{1}, \widehat{P}_{2}, \xi\right)+\cdots+\mu L_{1}^{\nu}\left(\widehat{P}_{1}, \widehat{P}_{2}, \xi_{3}\right)\right) \widetilde{\varphi}=E \widetilde{\varphi} .
$$

Для операторов $\widehat{P}_{1}, \widehat{P}_{2}$ выполнено обычное коммутационное соотношение Гейзенберга $\left[\widehat{P}_{1}, \widehat{P}_{2}\right]=i \mu B$, поэтому $(1.25)$ - псевдодифференциальное уравнение с одной степенью свободы. Этот факт становится совсем прозрачным, если вместо симметричной калибровки при определении векторного потенциала выбрать калибровку 
Ландау. Тогда $A=\cdots$ и

$$
\widehat{P}_{1}=-i \mu \frac{\partial}{\partial x_{1}}, \quad \widehat{P}_{2}=-i \mu \frac{\partial}{\partial x_{2}}+B x_{1}, \quad \widehat{P}_{3}=-i \mu \frac{\partial}{\partial x_{3}} .
$$

Тогда можно сделать преобразование Фурье по двум переменным $x_{2}, x_{3}$, и положить $z=x_{1}+\xi_{2} / B$. Тогда для соответствующей функции $\phi\left(z, \xi_{2}, \xi_{3}\right)$, где $\xi_{2}, \xi_{3}-$ переменные, двойственные к $x_{2}, x_{3}$, получаем уравнение

$$
\left(\mathscr{E} \nu\left(-i \mu \frac{\partial}{\partial z}, B \frac{1}{z}, \xi_{3}\right)+\mu L_{1}^{\nu}\left(-i \mu \frac{\partial}{\partial z}, B \stackrel{2}{z}, \xi_{3}\right)+\cdots+\mu L_{1}^{\nu}\left(-i \mu \frac{\partial}{\partial z}, B \stackrel{2}{z}, \xi_{3}\right)\right) \phi=E \phi .
$$

Приведенные соображения дают возможность перейти от квантового уравнения к уравнениям классической динамики. Для этого следует заменить в последнем уравнении оператор $-i \mu \partial / \partial z$ на классический импульс. Это дает классический гамильтониан с одной степенью свободы, зависящий от параметров. Если ввести соответствующую скобку Пуассона, то гамильтонову систему, отвечающую $\mathscr{E} \nu\left(\widehat{P}_{1}, \widehat{P}_{2}, \widehat{P}_{3}\right)$ (и "подправленному" гамильтониану (1.19)), можно написать сразу для классических длинных импульсов $P_{j}$. Ее траектории устроены весьма сложно, им посвящены очень серьезные исследования, как физиков, так и математиков. Наиболее полное изложение результатов и библиографию можно найти в [10].

\section{2. Метод стационарной фазы для осцилляторных интегралов с квад-} ратичной фазой. Рассмотрим интеграл

$$
F(h, \varphi)=\frac{1}{(\pi h)^{n / 2}} \int e^{i A x \cdot x / h} \varphi(x) d x
$$

где $h>0, x \in \mathbb{R}^{n}$, интегрирование производится по всему $\mathbb{R}^{n}, A$ - симметрическая вещественная невырожденная матрица. Если $\varphi(x) \in C_{0}^{\infty}\left(\mathbb{R}^{n}\right)$, то хорошо известный метод стационарной фазы утверждает, что при $h \rightarrow 0$ для $F(h, \varphi)$ имеет место асимптотическое разложение

$$
\left.F(h, \varphi) \sim|\operatorname{det} A|^{-1 / 2} e^{i \pi \sigma / 4} \sum_{j=0}^{\infty} \frac{1}{j !}\left(-i \frac{h}{4} A^{-1} D_{x} \cdot D_{x}\right)^{j} \varphi(x)\right|_{x=0},
$$

где $\sigma$ - сигнатура матрицы $A$, через $D_{x}$ обозначается

$$
D_{x}=-i \partial_{x}=\left(-i \partial_{x_{1}}, \ldots,-i \partial_{x_{n}}\right)
$$

(см., например, [11], [12]). В этом пункте статьи мы получим аналогичное разложение с оценкой остаточного члена для случая, когда функция $\varphi(x)$ принадлежит пространству $S^{m}$ из определения 1.1 (переменные $P$ в этом определении следует заменить на $x$ ). При этом интеграл в $(2.1)$, вообще говоря, не является сходящимся в обычном смысле, и его нужно определять специальным образом. Подобного рода интегралы часто встречаются в теории уравнений с частными производными, и их принято называть осцилляторными интегралами (см., например, [11]-[13]). Строгое определение интеграла в $(2.1)$ для $\varphi(x) \in S^{m}$ будет дано ниже.

Вернемся к случаю $\varphi(x) \in C_{0}^{\infty}\left(\mathbb{R}^{n}\right)$. Нам понадобится оценка остаточного члена в разложении $(2.2)$. Обозначим через $F_{N}(h, \varphi)$ разность между $F(h, \varphi)$ и правой частью формулы $(2.2)$, в которой суммирование производится по $0 \leqslant j<N$ (при этом 
$\left.F_{0}(h, \varphi)=F(h, \varphi)\right)$. Пусть носитель функции $\varphi(x)$ находится в ограниченной области $\Omega$ (т.е. $\varphi(x) \in C_{0}^{\infty}(\Omega)$ ). Тогда с некоторыми константами $C_{N}$ и $k_{N}$, зависящими только от области $\Omega$ и матрицы $A$, выполняется оценка

$$
\left|F_{N}(h, \varphi)\right| \leqslant C_{N} h^{N} \sum_{|\alpha| \leqslant k_{N}} \max \left|\varphi^{(\alpha)}(x)\right| .
$$

В таком виде это неравенство имеется в [12], в [11], [13] имеются более точные оценки, в частности, за $k_{N}$ можно взять $s_{n}+2 N$, где $s_{n}-$ наименьшее целое $>n / 2$ (см. [11; формула (7.7.7)]). Для полноты напомним схему вывода разложения (2.2) и оценки (2.3). Взяв за $f(x)$ функцию $\exp (i A x \cdot x / h)$, воспользуемся формулой преобразования Фурье такой функции:

$$
(\pi h)^{n / 2}|\operatorname{det} A|^{-1 / 2} e^{i \pi \sigma / 4} e^{-i h A^{-1} \xi \cdot \xi / 4}
$$

(см. [11], [12]). Тогда, исходя из соотношения

$$
\langle f(x), \varphi(x)\rangle=(2 \pi)^{-n}\langle\widetilde{f}(-\xi), \widetilde{\varphi}(\xi)\rangle, \quad f(x) \in S^{\prime}\left(\mathbb{R}^{n}\right), \quad \varphi(x) \in S\left(\mathbb{R}^{n}\right),
$$

получим

$$
F(h, \varphi)=\frac{1}{(\pi h)^{n / 2}}\langle f(x), \varphi(x)\rangle=(2 \pi)^{-n}|\operatorname{det} A|^{-1 / 2} e^{i \pi \sigma / 4} \int e^{-i h A^{-1} \xi \cdot \xi / 4} \widetilde{\varphi}(\xi) d \xi .
$$

Из этой формулы видно, что функция $F(h, \varphi)$, доопределенная по непрерывности при $h=0$, бесконечно дифференцируема при $h \geqslant 0$ и формула $(2.2)$ есть просто разложение функции $F(h, \varphi)$ в ряд Тейлора при $h=0$.

Разложение (2.2) и оценка (2.3) получаются разложением экспоненты в (2.4) в ряд Тейлора с применением неравенства

$$
\left|e^{i t}-\sum_{j=0}^{N-1} \frac{(i t)^{j}}{j !}\right| \leqslant t^{N}, \quad t \in \mathbb{R}^{1} .
$$

Учитывая, что при преобразовании Фурье оператор $D_{x}$ переходит в умножение на $\xi$, формулу (2.4) можно записывать в виде

$$
F(h, \varphi)=\left.|\operatorname{det} A|^{-1 / 2} e^{i \pi \sigma / 4} e^{-i h A^{-1} D_{x} \cdot D_{x} / 4} \varphi(x)\right|_{x=0},
$$

что очень удобно для запоминания разложения (2.2), поскольку правая часть в (2.2) получается формальным разложением экспоненты в (2.5) в ряд Тейлора.

Наша задача состоит в том, чтобы получить разложение $(2.2)$ для $\varphi(x) \in S^{m}$. Сначала рассмотрим интеграл $(2.1)$ в случае, когда $\varphi(x) \in C_{0}^{\infty}\left(\mathbb{R}^{n}\right)$ и $\varphi(x)=0$ в некоторой окрестности начала координат, например, $\varphi(x)=0$ в шаре $|x|<1$. Как тоже хорошо известно, для таких функций выполняются неравенства $|F(h, \varphi)| \leqslant$ $C_{N} h^{N}$ (как обычно, константы, которые обозначаются буквами $C$, в каждом новом неравенстве принимают новые значения). Напомним хорошо известный вывод таких неравенств, чтобы уточнить характер зависимости констант $C_{N}$ от функции $\varphi(x)$.

Обозначим через $S(x)$ функцию $S(x)=A x \cdot x$ и определим дифференциальный оператор

$$
L=\left|S_{x}^{\prime}\right|^{-2} \sum_{j=1}^{n} \frac{\partial S}{\partial x_{j}} \frac{\partial}{\partial x_{j}}
$$


В таком случае имеет место равенство

$$
L e^{i S(x) / h}=\frac{i}{h} e^{i S(x) / h} .
$$

Интегрируя по частям, получим

$$
\begin{aligned}
\int_{|x| \geqslant 1} \varphi(x) e^{i S(x) / h} d x & =\frac{h}{i} \int_{|x| \geqslant 1} \varphi(x) L\left(e^{i S(x) / h}\right) d x \\
& =\frac{h}{i} \int_{|x| \geqslant 1} e^{i S(x) / h}\left({ }^{t} L(\varphi(x))\right) d x
\end{aligned}
$$

где ${ }^{t} L$ - формально сопряженный оператор. Таким образом,

$$
F(h, \varphi)=-i h F\left(h,{ }^{t} L \varphi\right) .
$$

Повторяя такие интегрирования $k$ раз, получаем тождество

$$
F(h, \varphi)=(-i h)^{k} F\left(h,\left({ }^{t} L\right)^{k} \varphi\right) .
$$

Так как коэффициенты оператора $L$ являются однородными функциями порядка -1 , то при $|x|>1$

$$
\left|\left({ }^{t} L\right)^{k} \varphi(x)\right| \leqslant \frac{C_{k}}{|x|^{k}} \sum_{|\alpha| \leqslant k}\left|\varphi^{(\alpha)}(x)\right| .
$$

Пользуясь этим неравенством и тем, что $|x|$ и $1+|x|$ при $|x| \geqslant 1$ эквивалентны, получаем оценку

$$
|F(h, \varphi)| \leqslant h^{k}(\pi h)^{-n / 2} \max _{|x| \geqslant 1}\left|(1+|x|)^{n+1}\left({ }^{t} L\right)^{k}(\varphi(x))\right| \int \frac{d x}{(1+|x|)^{n+1}},
$$

следовательно,

$$
|F(h, \varphi)| \leqslant C_{k} h^{k-n / 2}\|\varphi(x)\|_{k, k-n-1}
$$

с другими константами $C_{k}$, причем константы $C_{k}$ не зависят от функции $\varphi(x) \in$ $C_{0}^{\infty}\left(\mathbb{R}_{x}^{n}\right)$ с носителем вне шара $|x|<1$. Нормы $\|\cdot\|_{k, m}$ определяются формулой $(1.21)$, в которой $P$ следует заменить на $x$. Зафиксируем некоторое целое $m \geqslant 0$. Поскольку $\|\varphi\|_{k, m_{1}} \leqslant\|\varphi\|_{k, m}$ при $m_{1} \geqslant m$, то

$$
|F(h, \varphi)| \leqslant C_{k} h^{k-n / 2}\|\varphi\|_{k, m}
$$

при $k-n-1 \geqslant m$. Пусть теперь задано некоторое целое число $N \geqslant 0$. Выбрав целое число $k_{N}^{\prime}$ так, чтобы выполнялись неравенства $k_{N}^{\prime} \geqslant m+n+1$ и $N \leqslant k_{N}^{\prime}-n / 2$, из (2.7) получим окончательную оценку

$$
|F(h, \varphi)| \leqslant C_{N} h^{N}\|\varphi\|_{k_{N}^{\prime}, m}
$$

при $0<h \leqslant 1$. Константа $C_{N}$ в $(2.8)$ равна константе $C_{k_{N}^{\prime}}$ из неравенства $(2.7)$.

Оценками (2.8) с произвольными $N$ мы будем пользоваться в дальнейшем при обсуждении асимптотики интеграла (2.1), а сейчас перейдем к определению осцилляторного интеграла (2.1) для произвольной амплитуды $\varphi(x) \in S^{m}$, где $m$ - неотрицательное целое число. Выберем срезающую функцию $\chi_{1}(x) \in C_{0}^{\infty}\left(\mathbb{R}^{n}\right)$, равную 
единице в шаре $|x|<1$. Пусть сначала $\varphi(x) \in C_{0}^{\infty}\left(\mathbb{R}^{n}\right)$. Разобьем функцию $\varphi(x)$ на два слагаемых

$$
\varphi(x)=\varphi_{1}(x)+\varphi_{2}(x), \quad \text { где } \quad \varphi_{1}(x)=\chi_{1}(x) \varphi(x), \quad \varphi_{2}(x)=\left(1-\chi_{1}(x)\right) \varphi(x) .
$$

Применив к функциям $\varphi_{1}(x)$ и $\varphi_{2}(x)$ неравенства $(2.3)$ и $(2.8)$ соответственно при $N=0$, получаем оценку вида

$$
|F(h, \varphi)| \leqslant C_{1} \sum_{|\alpha| \leqslant k_{0}} \max \left|\varphi_{1}^{(\alpha)}(x)\right|+C_{2}\left\|\varphi_{2}\right\|_{k_{0}^{\prime}, m} \leqslant C_{3}\left(\|\varphi\|_{k_{0}, m}+\|\varphi\|_{k_{0}^{\prime}, m}\right),
$$

из которой следует, что при любом целом $m \geqslant 0$ при фиксированном $h$ функционал $F(h, \varphi)$, определенный на $C_{0}^{\infty}\left(\mathbb{R}^{n}\right)$, ограничен по норме $\|\cdot\|_{k_{0}^{\prime \prime}, m}$ пространства $S^{m}$, где $k_{0}^{\prime \prime}=\max \left(k_{0}, k_{0}^{\prime}\right)$. Для произвольной $\varphi(x) \in S^{m}$ последовательность функций $\chi(\varepsilon x) \varphi(x)$, где $\chi(x) \in C_{0}^{\infty}\left(\mathbb{R}^{n}\right)$ и $\chi(x)$ равна единице в окрестности начала координат, сходится в $S^{m+1}$ к $\varphi(x)$. Отсюда следует, что существует предел $F(h, \chi(\varepsilon x) \varphi(x))$ при $\varepsilon \rightarrow 0$, который не зависит от выбора функции $\chi(x)$. Этот предел мы и будем обозначать как $F(h, \varphi)$. Тем самым, мы приходим к следующему определению.

ОПРЕДЕЛЕНиЕ 2.1. Значение осцилляторного интеграла в правой части (2.1) для любой функции $\varphi(x) \in S^{m}$ определяется с помощью равенства

$$
\int e^{i A x \cdot x / h} \varphi(x) d x=\lim _{\varepsilon \rightarrow 0} \int e^{i A x \cdot x / h} \chi(\varepsilon x) \varphi(x) d x .
$$

С другой стороны, если $\varphi(x) \in C_{0}^{\infty}\left(\mathbb{R}^{n}\right)$, то, используя тождество (2.6) для функции $\varphi_{2}(x)$, мы получим, что

$$
F(h, \varphi(x))=F\left(h, \chi_{1}(x) \varphi(x)\right)+(-i h)^{k} F\left(h,\left({ }^{t} L\right)^{k}\left(\left(1-\chi_{1}(x)\right) \varphi(x)\right)\right) .
$$

При $k \geqslant m+n+1$ интегралы в правой части этого равенства абсолютно сходятся для любых функций из $\varphi(x) \in S^{m}$, что видно из оценок, производившихся при доказательстве неравенства (2.8). Более того, заменив $\varphi(x)$ в равенстве $(2.9)$ на $\chi(\varepsilon x) \varphi(x)$ и переходя к пределу при $\varepsilon \rightarrow 0$, получим, что $(2.9)$ выполняется для всех $\varphi(x) \in S^{m}$, если $F(h, \varphi(x))$ понимать в смысле определения 1.1. Отсюда, в частности, следует, что правая часть в (2.9) не зависит от выбора $k$ при $k \geqslant m+n+1$, хотя это можно было бы доказать непосредстенно с помощью интегрирования по частям. Тем самым, мы приходим ко второму определению осцилляторного интеграла.

ОПРЕДЕЛЕНИЕ 2.2. Осцилляторный интеграл в правой части равенства (2.1) для $\varphi(x) \in S^{m}$ определяется равенством (2.9) при $k \geqslant m+n+1$, результат не зависит от выбора целого числа $k \geqslant m+n+1$ и функции $\chi_{1}(x) \in C_{0}^{\infty}\left(\mathbb{R}^{n}\right)$, равной единице в некоторой окрестности начала координат.

Из предыдущих рассуждений следует, что определения 2.1 и 2.2 эквивалентны. Теперь мы можем перейти к доказательству основного утверждения этого пункта.

Теорема 2.1. Если $\varphi(x) \in S^{m}$, то для $F(h, \varphi)$ в (2.1) имеет место асимптотическое разложение $(2.2)$, для остаточного члена при $0<h \leqslant 1$ выполняется оценка

$$
\left|F_{N}(h, \varphi(x))\right| \leqslant C_{N} h^{N}\|\varphi\|_{k_{N}^{\prime \prime}, m}
$$

с некоторыми константами $C_{N}$ и $k_{N}^{\prime \prime}$, не зависящими от функиии $\varphi$. 
ДоКАЗАТЕЛЬСТвО этого утверждения состоит в том, что мы опять разбиваем функцию $\varphi(x)$ на сумму двух функций

$$
\varphi_{1}(x)=\chi_{1}(x) \varphi(x) \quad \text { и } \quad \varphi_{2}(x)=\left(1-\chi_{1}(x)\right) \varphi(x),
$$

для первой пользуемся оценкой (2.3), а для второй - оценкой (2.8).

В завершение пункта рассмотрим поведение осциллятороного интеграла (2.1), когда функция $\varphi$ зависит от дополнительных параметров $y \in \mathbb{R}^{n}$. Будем предполагать, что $\varphi(x, y)$ принадлежит $S^{m}\left(\mathbb{R}_{x}^{n} \times \mathbb{R}_{y}^{n}\right)$, т.е. для любых мультииндексов $\alpha$ и $\beta$ выполняются неравенства

$$
\left|\partial_{x}^{\alpha} \partial_{y}^{\beta} \varphi(x, y)\right| \leqslant C_{\alpha, \beta}(1+|x|+|y|)^{m}
$$

В дальнейшем будем считать, что $m$ - целое неотрицательное число. Так как выполняются неравенства

$$
\left(1+|x|^{m}+|y|^{m}\right) \leqslant(1+|x|+|y|)^{m} \leqslant C_{m}\left(1+|x|^{m}+|y|^{m}\right),
$$

то (2.11) эквивалентны неравенствам

$$
\left|\partial_{x}^{\alpha} \partial_{y}^{\beta} \varphi(x, y)\right| \leqslant C_{\alpha, \beta}\left(1+|x|^{m}+|y|^{m}\right)
$$

с другими константами $C_{\alpha, \beta}$. Неравенства $(2.12)$ являются следствием более общего неравенства

$$
\left(|z|^{m}+|x|^{m}+|y|^{m}\right) \leqslant C_{m}\left(|z|^{m}+|x|^{m}+|y|^{m}\right)
$$

которое вытекает из того, что левая и правая части этого неравенства - это однородные функции одинаковых порядков однородности и отличны от нуля при $|x|+|y|+|z| \neq 0$.

В силу того, что функция $\varphi(x, y)$ при фиксированном $y$ принадлежит $S^{m}\left(\mathbb{R}_{x}^{n}\right)$, с помощью формулы (2.1) определяется функция

$$
\Phi(h, y)=F(h, \varphi(x, y))
$$

(в формуле $(2.1)$ нужно $\varphi(x)$ заменить на $\varphi(x, y)$ ).

ТЕОРЕма 2.2. Если для $\varphi(x, y)$ выполнены неравенства $(2.11)$ с целым $m \geqslant 0$, то функиия $\Phi(h, y)$ принадлежит $S^{m}\left(\mathbb{R}_{y}^{n}\right)$ и допускает асимптотическое разложение $(2.2)$, в котором $\left.\varphi(x)\right|_{x}=0$ нужно заменить на $\left.\varphi(x, y)\right|_{x}=0$, а остаточный член $\Phi_{N}(h, y)$ имеет оценку

$$
\left|\partial_{y}^{\beta} \Phi_{N}(h, y)\right| \leqslant C_{N, \beta} h^{N}(1+|y|)^{m}
$$

при любъх $N$ u $\beta, 0<h \leqslant 1$.

ДокАЗАтЕЛьСтво. Заметим, что

$$
\partial_{y}^{\beta} \Phi_{N}(h, y)=F_{N}\left(h, \partial_{y}^{\beta} \varphi(x, y)\right) .
$$

Пользуясь неравенствами (2.10) и (2.13), получим

$$
\begin{aligned}
\left|\partial_{y}^{\beta} \Phi_{N}(h, y)\right| & \leqslant C_{N} h^{N} \sum_{|\alpha| \leqslant k_{N}^{\prime \prime}} \sup _{x} \frac{\left|\partial_{x}^{\alpha} \partial_{y}^{\beta} \varphi(x, y)\right|}{(1+|x|)^{m}} \\
& \leqslant C_{N} h^{N} \sum_{|\alpha| \leqslant k_{N}^{\prime \prime}} C_{\alpha, \beta} \sup _{x} \frac{1+|x|^{m}+|y|^{m}}{(1+|x|)^{m}}
\end{aligned}
$$

откуда и следуют неравенства (2.14). 
3. Формула композиции. Положим

$\widehat{P}_{1}=h D_{x_{1}}+q_{12} x_{2}+q_{13} x_{3}, \quad \widehat{P}_{2}=h D_{x_{2}}+q_{21} x_{1}+q_{23} x_{3}, \quad \widehat{P}_{3}=h D_{x_{3}}+q_{31} x_{1}+q_{32} x_{2}$, где $D_{x_{k}}=-i \partial_{x_{k}}, h$ - положительная постоянная, $q_{k j}$ в этом пункте - это произвольные вещественные постоянные. Пусть задана функция $a\left(\eta_{1}, \eta_{2}, \eta_{3}\right)$. Определим по $a\left(\eta_{1}, \eta_{2}, \eta_{3}\right)$ оператор $a\left(\widehat{P}_{1}, \widehat{P}_{2}, \widehat{P}_{3}\right)$, причем будем считать, что $\widehat{P}_{1}$ действует первым, $\widehat{P}_{2}$ действует вторым, $\widehat{P}_{3}$ - третьим в смысле операторного исчисления из книги Маслова [4]. Функцию $a\left(\eta_{1}, \eta_{2}, \eta_{3}\right)$ называют символом такого оператора. Будем рассматривать только символы, которые принадлежат классу $S^{m}\left(\mathbb{R}_{\eta}^{3}\right)$. Аналогично обычным псевдодифференциальным операторам, $a\left(\widehat{P}_{1}, \widehat{P}_{2}, \widehat{P}_{3}\right)$ можно рассматривать как $h$-псевдодифференциальный оператор вида

$$
\begin{aligned}
& a\left(\widehat{P}_{1}, \widehat{P}_{2}, \widehat{P}_{3}\right) u(x) \\
& =\frac{1}{(2 \pi h)^{3}} \int a\left(\xi_{1}+q_{12} y_{2}+q_{13} y_{3}, \xi_{2}+q_{21} x_{1}+q_{23} y_{3}, \xi_{3}+q_{31} x_{1}+q_{32} x_{2}\right) \\
& \quad \times e^{i(x-y) \cdot \xi / h} u(y) d y d \xi .
\end{aligned}
$$

Интеграл в (3.1) следует понимать как осцилляторный интеграл в смысле определений 2.1 и 2.2 , причем интегрирование производится по всему пространству $\mathbb{R}_{y}^{3} \times \mathbb{R}_{\xi}^{3}$. Оператор $a\left(\widehat{P}_{1}, \widehat{P}_{2}, \widehat{P}_{3}\right)$ можно записать в виде

$$
a\left(\widehat{P}_{1}, \widehat{P}_{2}, \widehat{P}_{3}\right) u(x)=\frac{1}{(2 \pi h)^{3}} \int e^{-i M(x, y) / h+i(x-y) \cdot \eta / h} a(\eta) u(y) d y d \eta,
$$

где

$M(x, y)=\left(x_{1}-y_{1}\right)\left(q_{12} y_{2}+q_{13} y_{3}\right)+\left(x_{2}-y_{2}\right)\left(q_{21} x_{1}+q_{23} y_{3}\right)+\left(x_{3}-y_{3}\right)\left(q_{31} x_{1}+q_{32} x_{2}\right)$,

или в виде

$$
a\left(\widehat{P}_{1}, \widehat{P}_{2}, \widehat{P}_{3}\right) u(x)=\int K_{a}(x, y, h) u(y) d y,
$$

где ядро интегрального оператора (3.4) определяется осцилляторным интегралом

$$
K_{a}(x, y, h)=\frac{1}{(2 \pi h)^{3}} e^{-i M(x, y) / h} \int e^{i(x-y) \cdot \eta / h} a(\eta) d \eta .
$$

Переходя от $y$ к $t=x-y$, получим, что обобщенная функция

$$
K_{a}(x, x-t, h) \exp \left(i h^{-1} M(x, x-t)\right)
$$

есть обратное $h$-преобразование Фурье от символа $a(\eta)$, следовательно, не зависит от $x$, а сам символ $a(\eta)$ восстанавливается с помощью формулы обращения $h$-преобразования Фурье:

$$
\begin{aligned}
a(\eta) & =\int e^{-i t \cdot \eta / h} K_{a}(x, x-t, h) e^{i M(x, x-t) / h} d t \\
& =\int e^{-i(x-y) \eta} / h K_{a}(x, y, h) e^{i M(x, y) / h} d y .
\end{aligned}
$$

Наша задача - изучить композицию (т.е. произведение)

$$
a_{2}\left(\widehat{P}_{1}, \widehat{P}_{2}, \widehat{P}_{3}\right) a_{1}\left(\widehat{P}_{1}, \widehat{P}_{2}, \widehat{P}_{3}\right)
$$


операторов с символами из $S^{m_{2}}$ и $S^{m_{1}}$ соответственно. Будем считать, что $m_{2} \geqslant 0$ и $m_{1} \geqslant 0$. Мы покажем, что такая композиция сама является оператором с символом (зависящим от $h$ ), который обозначим как $b\left(\zeta_{1}, \zeta_{2}, \zeta_{3}, h\right)$, и дадим асимптотическое разложение этого символа по параметру $h \rightarrow 0$.

Теорема 3.1. Символ композиции $a_{2}\left(\widehat{P}_{1}, \widehat{P}_{2}, \widehat{P}_{3}\right) a_{1}\left(\widehat{P}_{1}, \widehat{P}_{2}, \widehat{P}_{3}\right)$ имеет асимптотическое разложение

$$
b(\zeta, h)=\sum \frac{\mu^{k_{1}+k_{2}+k_{3}}}{k_{1} ! k_{2} ! k_{3} !}\left(\left(-i \widetilde{q}_{12} \partial_{\zeta_{1}}\right)^{k_{1}}\left(-i \widetilde{q}_{13} \partial_{\zeta_{1}}\right)^{k_{2}}\left(-i \widetilde{q}_{23} \partial_{\zeta_{2}}\right)^{k_{3}} a_{2}(\zeta)\right) \partial_{\zeta_{2}}^{k_{1}} \partial_{\zeta_{3}}^{k_{2}} \partial_{\zeta_{3}}^{k_{3}} a_{1}(\zeta)
$$

где суммирование производится по всем иелым неотрицательным $k_{1}, k_{2}, k_{3} u$ $\widetilde{q}_{j k}=q_{k j}-q_{j k}$. Остаточный член $b_{N}(\zeta, h)$, который получается при учете в сумме (3.6) только членов с $k_{1}+k_{2}+k_{3}<N$, является величиной порядка $O\left(h^{N}\right)$ относительно $S^{m}$ с $m=m_{1}+m_{2}$, причем для любых иелых $N \geqslant 0, k \geqslant 0, m \geqslant 0$ найдутся такие челье числа $k_{1} \geqslant 0 u k_{2} \geqslant 0$, что при $0<h \leqslant 1$ выполняются оценки

$$
\left\|b_{N}(\zeta, h)\right\|_{k, m} \leqslant C_{N, k, m} h^{N}\left\|a_{1}\right\|_{k_{1}, m}\left\|a_{2}\right\|_{k_{2}, m} .
$$

ДокАЗАТЕЛЬСтво. Используя формулу (3.2), запишем $v=a_{1}\left(\widehat{P}_{1}, \widehat{P}_{2}, \widehat{P}_{3}\right) u$ в виде

$$
v(z)=\frac{1}{(2 \pi h)^{3}} \int e^{-i M(z, y) / h+i(z-y) \cdot \eta / h} a_{1}(\eta) u(y) d y d \eta .
$$

Тогда в силу формулы (3.2)

$$
\begin{aligned}
& a_{2}\left(\widehat{P}_{1}, \widehat{P}_{2}, \widehat{P}_{3}\right) a_{1}\left(\widehat{P}_{1}, \widehat{P}_{2}, \widehat{P}_{3}\right) u \\
& \quad=a_{2}\left(\widehat{P}_{1}, \widehat{P}_{2}, \widehat{P}_{3}\right) v=\frac{1}{(2 \pi h)^{6}} \int e^{i(-M(x, z)-M(z, y)+L) / h} a_{2}(\theta) a_{1}(\eta) u(y) d y d \eta d z d \theta,
\end{aligned}
$$

где $L=(z-y) \cdot \eta+(x-z) \cdot \theta$. Чтобы получить символ $b(\zeta, h)$, мы используем для $b$ формулу, аналогичную (3.5):

$$
\begin{aligned}
\int e^{i(x-y) \cdot \zeta / h} b(\zeta, h) d \zeta & =(2 \pi h)^{3} e^{i M(x, y) / h} K_{b}(x, y, h) \\
& =\frac{1}{(2 \pi h)^{3}} \int e^{i(M(x, y)-M(x, z)-M(z, y)+L) / h} a_{2}(\theta) a_{1}(\eta) d \eta d z d \theta
\end{aligned}
$$

Конечно, предварительно нужно проверить, что правая часть в (3.8) является функцией от $x-y$.

Приведем к более простому виду показатель экспоненты в (3.8). Раскрывая скобки в выражениях вида (3.3) и группируя подобные члены, нетрудно получить, что

$$
M(x, y)-M(x, z)-M(z, y)=-\widetilde{q}_{12} \widetilde{z}_{1}\left(z_{2}-y_{2}\right)-\widetilde{q}_{13} \widetilde{z}_{1} \widetilde{z}_{3}-\widetilde{q}_{23} \widetilde{z}_{3}\left(z_{2}-x_{2}\right),
$$

где

$$
\tilde{q}_{i k}=q_{k j}-q_{j k}, \quad \widetilde{z}_{1}=z_{1}-x_{1}, \quad \widetilde{z}_{3}=z_{3}-y_{3},
$$

a $L=(z-y) \cdot \eta+(x-z) \cdot \theta$ приводится к виду

$$
L=\left(x_{1}-y_{1}\right) \eta_{1}+\widetilde{z}_{1}\left(\eta_{1}-\theta_{1}\right)+\left(x_{2}-z_{2}\right) \theta_{2}+\left(z_{2}-y_{2}\right) \eta_{2}+\left(x_{3}-y_{3}\right) \theta_{3}+\widetilde{z}_{3}\left(\eta_{3}-\theta_{3}\right) .
$$


Переходя от переменных $x, y$ к переменным $t=x-y, t^{\prime}=x+y$ (обратная замена: $\left.x=\left(t+t^{\prime}\right) / 2, y=\left(t^{\prime}-t\right) / 2\right)$, запишем слагаемые в $L$, относящиеся к координатам с индексом 2 , в виде

$$
\left(x_{2}-z_{2}\right) \theta_{2}+\left(z_{2}-y_{2}\right) \eta_{2}=\frac{t_{2}}{2}\left(\theta_{2}+\eta_{2}\right)+\frac{t_{2}^{\prime}}{2}\left(\theta_{2}-\eta_{2}\right)+z_{2}\left(\eta_{2}-\theta_{2}\right) .
$$

Обозначая

$$
\widetilde{\theta}_{1}=\theta_{1}-\eta_{1}, \quad \widetilde{\eta}_{3}=\eta_{3}-\theta_{3}
$$

запишем $L$ в виде

$$
L=\left(x_{1}-y_{1}\right) \eta_{1}-\widetilde{z}_{1} \widetilde{\theta}_{1}+\left(x_{3}-y_{3}\right) \theta_{3}+\widetilde{z}_{3} \widetilde{\eta}_{3}+\frac{t_{2}}{2}\left(\theta_{2}+\eta_{2}\right)+\left(\frac{t_{2}^{\prime}}{2}-z_{2}\right)\left(\theta_{2}-\eta_{2}\right) .
$$

В результате показатель экспоненты в (3.8) приводится к

$$
\begin{aligned}
& \frac{i}{h}\left[-\widetilde{q}_{13} \widetilde{z}_{1} \widetilde{z}_{3}-\left(z_{2}-\frac{t^{\prime}}{2}\right)\left(\widetilde{q}_{12} \widetilde{z}_{1}+\widetilde{q}_{23} \widetilde{z}_{3}+\theta_{2}-\eta_{2}\right)+\right. \\
& \left.\quad+\frac{t_{2}}{2}\left(\theta_{2}+\eta_{2}-\widetilde{q}_{12} \widetilde{z}_{1}+\widetilde{q}_{23} \widetilde{z}_{3}\right)-\widetilde{z}_{1} \widetilde{\theta}_{1}+\widetilde{z}_{3} \widetilde{\eta}_{3}+t_{1} \eta_{1}+t_{3} \theta_{3}\right] .
\end{aligned}
$$

Теперь ясно, что в интеграле (3.8) нужно сделать замену переменных: от интегрирования по $z, \theta, \eta$ перейти к интегрированию по переменным $\widetilde{z}_{1}, \widetilde{z}_{2}, \widetilde{z}_{3}, \widetilde{\theta}_{1}, \theta_{2}, \theta_{3}$, $\eta_{1}, \eta_{2}, \widetilde{\eta}_{3}$, где $\widetilde{z}_{2}=z_{2}-t_{2}^{\prime} / 2$, а остальные переменные определены формулами (3.9), (3.10), затем перейти к новым переменным, заменив $\theta_{2}$ и $\eta_{2}$ на

$$
s_{1}=\widetilde{q}_{12} \widetilde{z}_{1}+\widetilde{q}_{23} \widetilde{z}_{3}+\theta_{2}-\eta_{2}, \quad s_{2}=\frac{\widetilde{q}_{23} \widetilde{z}_{3}-\widetilde{q}_{12} \widetilde{z}_{1}+\theta_{2}+\eta_{2}}{2},
$$

причем

$$
\theta_{2}=\frac{s_{1}}{2}+s_{2}-\widetilde{q}_{23} \widetilde{z}_{3}, \quad \eta_{2}=s_{2}-\frac{s_{1}}{2}+\widetilde{q}_{12} \widetilde{z}_{1} .
$$

Обозначим через $A$ квадратичную форму

$$
A\left(\widetilde{z}_{1}, \widetilde{z}_{3}, \widetilde{\theta}_{1}, \widetilde{\eta}_{3}\right)=-\widetilde{q}_{13} \widetilde{z}_{1} \widetilde{z}_{3}-\widetilde{z}_{1} \widetilde{\theta}_{1}+\widetilde{z}_{3} \widetilde{\eta}_{3},
$$

тогда формула (3.8) приобретает вид

$$
\begin{aligned}
& \frac{1}{(2 \pi h)^{3}} \int e^{i\left(t_{1} \zeta_{1}+t_{2} \zeta_{2}+t_{3} \zeta_{3}\right) / h} b\left(\zeta_{1}, \zeta_{2}, \zeta_{3}, h\right) d \zeta_{1} \zeta_{2} d \zeta_{3} \\
& \quad=\frac{1}{(2 \pi h)^{6}} \int e^{i\left(A-\widetilde{z}_{2} s_{1}+t_{2} s_{2}+t_{1} \eta_{1}+t_{3} \theta_{3}\right) / h} a_{2}(\theta) a_{1}(\eta) d \widetilde{z} d \widetilde{\theta}_{1} d \eta_{1} d s_{1} d s_{2} d \theta_{3} d \widetilde{\eta}_{3},
\end{aligned}
$$

где $\theta$ и $\eta$ выражаются через переменные, по которым производится интегрирование, по формулам (3.10) и (3.11). Трехмерное $h$-преобразование Фурье в этом равенстве можно рассматривать как последовательное применение одномерных преобразований. Так в левой части имеется одномерное обратное $h$-преобразование Фурье от переменной $\zeta_{1}$ к $t_{1}$, а в правой - от $\eta_{1}$ к $t_{1}$, следовательно, на основании формулы обращения $h$-преобразования Фурье мы можем опустить интегрирования по $\zeta_{1}$ в левой и по $\eta_{1}$ в правой частях, заменив $\eta_{1}$ в правой части на $\zeta_{1}$. Аналогичным образом можно поступить и с интегрированиями по $\zeta_{3}$ в правой и по $\theta_{3}$ в левой 
частях этого равенства. Применив после этого одномерную формулу обращения $h$-преобразования Фурье по переменной $\zeta_{2}$ и переходя от $s_{2}$ к $\widetilde{s}_{2}=s_{2}-\zeta_{2}$, получим

$$
b\left(\zeta_{1}, \zeta_{2}, \zeta_{3}, h\right)=\frac{1}{(2 \pi h)^{4}} \int e^{i\left(A-\widetilde{z}_{2} s_{1}+t_{2} \widetilde{s}_{2}\right) / h} a_{2}(\theta) a_{1}(\eta) d \widetilde{z} d \widetilde{\theta}_{1} d s_{1} d \widetilde{s}_{2} d \widetilde{\eta}_{3} d t_{2} .
$$

Так как амплитуда $a_{2}(\theta) a_{1}(\eta)$ не зависит от $t_{2}$ и $\widetilde{z}_{2}$, то из формулы обращения $h$-преобразования Фурье следует, что можно опустить интегрирование по переменным $\widetilde{z}_{2}$, $s_{1}, t_{2}, \widetilde{s}_{2}$, добавив множитель $(2 \pi h)^{2}$ и положив $s_{1}=\widetilde{s}_{2}=0$ :

$$
\begin{aligned}
& b\left(\zeta_{1}, \zeta_{2}, \zeta_{3}, h\right) \\
& =\frac{1}{(2 \pi h)^{2}} \int e^{i A\left(\widetilde{z}_{1}, \widetilde{z}_{3}, \widetilde{\theta}_{1}, \widetilde{\eta}_{3}\right) / h} a_{2}\left(\widetilde{\theta}_{1}+\zeta_{1}, \zeta_{2}-\widetilde{q}_{23} \widetilde{z}_{3}, \zeta_{3}\right) \\
& \quad \times a_{1}\left(\zeta_{1}, \zeta_{2}+\widetilde{q}_{12} \widetilde{z}_{1}, \eta_{3}+\widetilde{\zeta}_{3}\right) d \widetilde{z}_{1} d \widetilde{z}_{3} d \widetilde{\theta}_{1} d \widetilde{\eta}_{3},
\end{aligned}
$$

Для завершения доказательства остается к этому интегралу применить метод стационарной фазы, сформулированный в теореме 2.2 . Подсчитав матрицу $B$, которая является обратной к матрице формы $A$, получим

$$
B=\left(\begin{array}{cccc}
0 & 0 & -2 & 0 \\
0 & 0 & 0 & 2 \\
-2 & 0 & 0 & -2 \widetilde{q}_{13} \\
0 & 2 & -2 \widetilde{q}_{13} & 0
\end{array}\right) .
$$

Записав теперь формулу (2.5) для нашего случая, получим, что символ $b$ может быть представлен в виде

$$
b(\zeta, h)=\left.\exp \left(i h\left(\widetilde{q}_{12} D_{\eta_{2}} D_{\theta_{1}}+\widetilde{q}_{13} D_{\eta_{3}} D_{\theta_{1}}+\widetilde{q}_{23} D_{\eta_{3}} D_{\theta_{2}}\right)\right) a_{2}(\theta) a_{1}(\eta)\right|_{\eta=\zeta, \theta=\zeta} .
$$

Разлагая экспоненту в ряд Тейлора, как и в п. 2 , мы как раз получим асимптотическое разложение (3.6). Остальные утверждения теоремы 3.1 следуют из теоремы 2.2 , примененной к интегралу (3.12). Теорема доказана.

ЗАмечАнИЕ 1 . Если хотя бы один из символов $a_{1}(\eta), a_{2}(\theta)$ является многочленом, то в разложении (3.6) остается лишь конечное число слагаемых, и (3.6) превращается из асимптотического в точное равенство (т.е. остаточный член равен нулю).

Это видно, если записать формулу (3.13) в виде, аналогичном формуле (2.4), понимая преобразование Фурье соответствующей функции $\varphi$ в смысле теории обобщенных функций.

ЗАмечАниЕ 2. Утверждение теоремы 3.1 остается справедливым, если символы $a_{1}$ и $a_{2}$ зависят также от $h$, причем они являются величинами порядка $O(1)$ по $h$ относительно $S^{m_{1}}$ и $S^{m_{2}}$ соответственно в смысле определения 1.1.

Доказательство этого утверждения ничем не отличается от доказательства теоремы 3.1. В теореме 3.1 мы рассматривали символы, не зависящие от $h$, чтобы не загромождать соответствующие формулы.

В частности, из этого утверждения следует, что объединение по всем $m$ операторов $a\left(\widehat{P}_{1}, \widehat{P}_{2}, \widehat{P}_{3}, h\right)$, с символами порядка $O(1)$ относительно $S^{m}$ является алгеброй.

ЗАмЕчаниЕ 3 . Если выполнены условия теоремы 3.1 , то символ $b(\zeta, h)$, доопределенный по непрерывности при $h=0$, является бесконечно дифференцируемой 
по совокупности переменных функцией при $h \geqslant 0, \zeta \in \mathbb{R}^{3}$, а для производных от $b_{N}(\zeta, h)$ по $h$ выполняются оценки вида (3.7) с константами $C_{N, k, m}$, зависящими от порядка такой производной.

Доказательство этого утверждения лишь с небольшими изменениями повторяет доказательство теоремы 3.1 .

Авторы благодарны В. Е. Назайкинскому за полезные обсуждения и советы.

\section{СПИСОК ЦИТИРОВАННОЙ ЛИТЕРАТУРЫ}

[1] Е. М. Лифшиц, Л. П. Питаевский, Теоретическая физика, т. 9, Статистическая физика, ч. 2, Теория конденсированного состояния, Наука, М., 1978.

[2] G. Panati, H. Spohn, S. Teufel, "Effective dynamics for Bloch electrons: Peierls substitution and beyond", Comm. Math. Phys., 242:3, (2003), 547-578.

[3] E. I. Blount, "Bloch electrons in a magnetic field", Phys. Rev. (2), 126:5, (1962), $1636-1653$.

[4] В. П. Маслов, Операторные методь, Наука, М., 1973.

[5] Л. В. Берлянд, С. Ю. Доброхотов, ““Юператорное разделение переменных” в задаче о коротковолновой асимптотике для дифференциальных уравнений с быстроменяющимися коэффициентами", Докл. АН СССР, 296:1, (1987), 80-84.

[6] V. V. Belov, S. Yu. Dobrokhotov, T. Ya. Tudorovskiy, "Operator separation of variables for adiabatic problems in quantum and wave mechanics", J. Engrg. Math., 55:1-4, (2006), $183-237$.

[7] В.В.Белов, С. Ю. Доброхотов, В. П. Маслов, Т. Я. Тудоровский, “Обобщенный адиабатический принцип для описания динамики электрона в искривленных наноструктурах", УФН, 175:9, (2005), 1004-1010.

[8] М.В. Карасёв, В. П. Маслов, “Алгебры с общими перестановочными соотношениями и их приложения. II. Операторные унитарно-нелинейные уравнения", Итоги науки и техн. Сер. Соврем. пробл. мат., 13, ВИНИТИ, М., 1979, 145-267.

[9] М.А. Шубин, Псевдодифференциальные операторь и спектральная теория, Наука, M., 1978.

[10] С. П. Новиков, А. Я. Мальцев, "Топологические явления в нормальных металлах", УФН, 168:3, (1998), 249-258.

[11] Л. Хёрмандер, Анализ линейных дифференииальрых операторов с частными производными. Т. 1. Теория распределений и анализ Фуръе, Мир, М., 1986.

[12] М. В. Федорюк, Асимптотика: интеграль и ряды, Справочная математическая библиотека, Наука, М., 1987.

[13] S. Albeverio, Z. Brzeźniak, "Finite dimensional approximation approach to oscillatory integrals and stationary phase in infinite dimensions", J. Funct. Anal., 113:1, (1993), $177-244$.

\section{В. В. Грушин}

Поступило

Московский государственный институт

16.10.2009

электроники и математики,

Московский физико-технический институт

(государственный университет)

E-mail: vvgrushin@mail.ru

\section{С. Ю. Доброхотов}

Институт проблем механики РАН,

Московский физико-технический институт

(государственный университет)

E-mail: dobr@ipmnet.ru 